\title{
Which Drivers of User Engagement Support the Cost-Effectiveness of Online Self-Help Programs? Amazon Turkers Tell Web Managers What Matters
}

By

Michiel Bosman, Oklahoma State University

Joan Swart, Open Forest LLC

$\Lambda_{\text {savings }}^{\text {ccess of }} \begin{gathered}\text { care, } \\ \text { cont }\end{gathered}$ convenience for both clients and healthcare professionals are but some of the reasons why there is a lot of interest in the development of effective, evidence-based telemental health services. Even more than in "classical" face-to-face therapy, online mental health programs ("telemental health" and/or online self-help programs) must focus on client retention. The
Open Forest is a provider of online mental health self-help programs and, in this context, it is crucial to understand the needs of potential users. With a high prevalence of ADHD, user needs to establish commitment and loyalty centered on the ability to engage with others online. online self-help mental health programs have huge efficiency and scalability potentials, but to be effective, clients will have to actively participate in these programs. To address the client retention issue, this study aims to replicate previous research in user commitment to User Generated Content (UGC) websites, and create a framework for enhancing user engagement in online mental health self-help websites.

Keywords: Healthcare Management, Self-Help, Social Exchange Theory, Social Penetration Theory, Amazon Mechanical Turk, Behavioral Research, Telemental Health

Copyright ( 2017 , Muma College of Business. This article is published under a Creative Commons BY-NC license. Permission is granted to copy and distribute this article for non-commercial purposes, in both printed and electronic formats 


\section{Introduction}

Access to care, purported cost savings and convenience for both clients and healthcare professionals are but some of the reasons why there is a lot of interest in the development of effective, evidence-based telemental health services. There is a considerable amount of research defining what types of interventions are (and are not) effective in face-to-face psychotherapy. The cognitive-behavioral therapy approach is one of the most widely researched approaches. Its models have been translated into online therapy versions, which have been shown to have a positive effect on mental health symptom reduction. There are four major types of telemental health services. The first type is a direct replacement for faceto-face contact (e.g., via videoconferencing). This type uses the internet mainly to create a secure voice or video communication channel between client and therapist. The second type is a hybrid version of "classical" face-to-face therapy (either on-site or via video conferencing) and a structured online therapy program (comparable to college e-learning modules). The third type replaces the face-to-face communication with online chat functionality. The fourth type is a pure online self-help therapy program (which might be best compared to e-learning modules--best known under the acronym MOOCs (Massive Open Online Courses).

Even more than in "classical" face-to-face therapy, online mental health programs must focus on client retention. Dropout rates are notoriously high and this significantly reduces the potential cost efficiency gained by offering programs online. The fourth type mentioned above (the online self-help therapy program) has huge efficiency and scalability potentials, but client retention is an essential factor to focus on.

While mental health experts (this paper will use the terms therapists, counselors, and psychotherapists) have been trained in the importance of evidence-based practice for their therapeutic endeavors, they have largely ignored the evidence available for client retention. A major reason for this was the purported importance of the client's intrinsic motivation for therapeutic success, and possibly a lack of awareness of the availability of evidence-based client retention methods. A major breakthrough in this field was the development of the technique of Motivational Interviewing in addiction medicine. Its premise was that it is nice to have a successful treatment strategy, but if the client drops out of treatment and relapses in addiction behavior, who cares about the theoretical possibility of success had the client continued treatment?

For the telemental health program with limited client-counselor interaction (types three and four described above), the "Motivational Interviewing" techniques have limited applicability, as those tech- niques depend heavily on client-counselor interaction. There is an abundance of research relevant to those programs, although most of this research is unpublished or at least not available in peer-reviewed publications. Measuring and perfecting ("tweaking") user experience is an essential part of the multi-billion-dollar experiment formed by the major internet companies. Altounian describes this in his 2014 dissertation. For User Generated Content (UGC) sites, "revenue streams are dependent on active bases of users and user-generated traffic. Creating conditions that encourage active, regular participation by users is a key objective of UGC website platform providers. Since UGC sites' platform providers supply the channel rather than the actual content, platform providers need to ensure that their websites appeal to both producers and consumers of the content" (Altounian, 2014). The difference between UGC sites and regular e-commerce sites is shown in Figure 1. While telemental health sites of types two through four may provide content, their "raison detre" is being a platform for user interaction. A telemental health client uses the content to produce positive health outcomes. As such, they are comparable to UGC sites more so than to regular e-commerce sites. This fits in with Van Dijck's findings that users of UGC sites can either be producers of content, passive consumers of content, or a combination of producer and consumer (Van Dijck, 2009).

In his dissertation research, Altounian used a combination of social exchange theory and social penetration theory "to propose a model for evaluating factors that lead to deeper commitment levels for a particular user generated content site. This model consists of four constructs related to users' perceptions of importance of site content, the community on that site, the specific characteristics of the site, and the perceived benefits to the user" (Altounian 2014).

He found that the User Benefit construct and the Content construct were the strongest predictors of User Commitment to a particular website.

As user commitment is such an important factor in user retention, we decided to test Altounian's model with regard to an online mental health self-help website. Our hypothesis is that Benefit and Commitment are predictors of user retention, and that this correlation is stronger for those with experience in mental health counselling.

\section{Review of Research}

\section{Online Therapy}

The delivery of psychotherapy and mental health self-help programs through Internet-based channels is a relatively new concept that has only really gained traction in the past decade. Prior studies have 


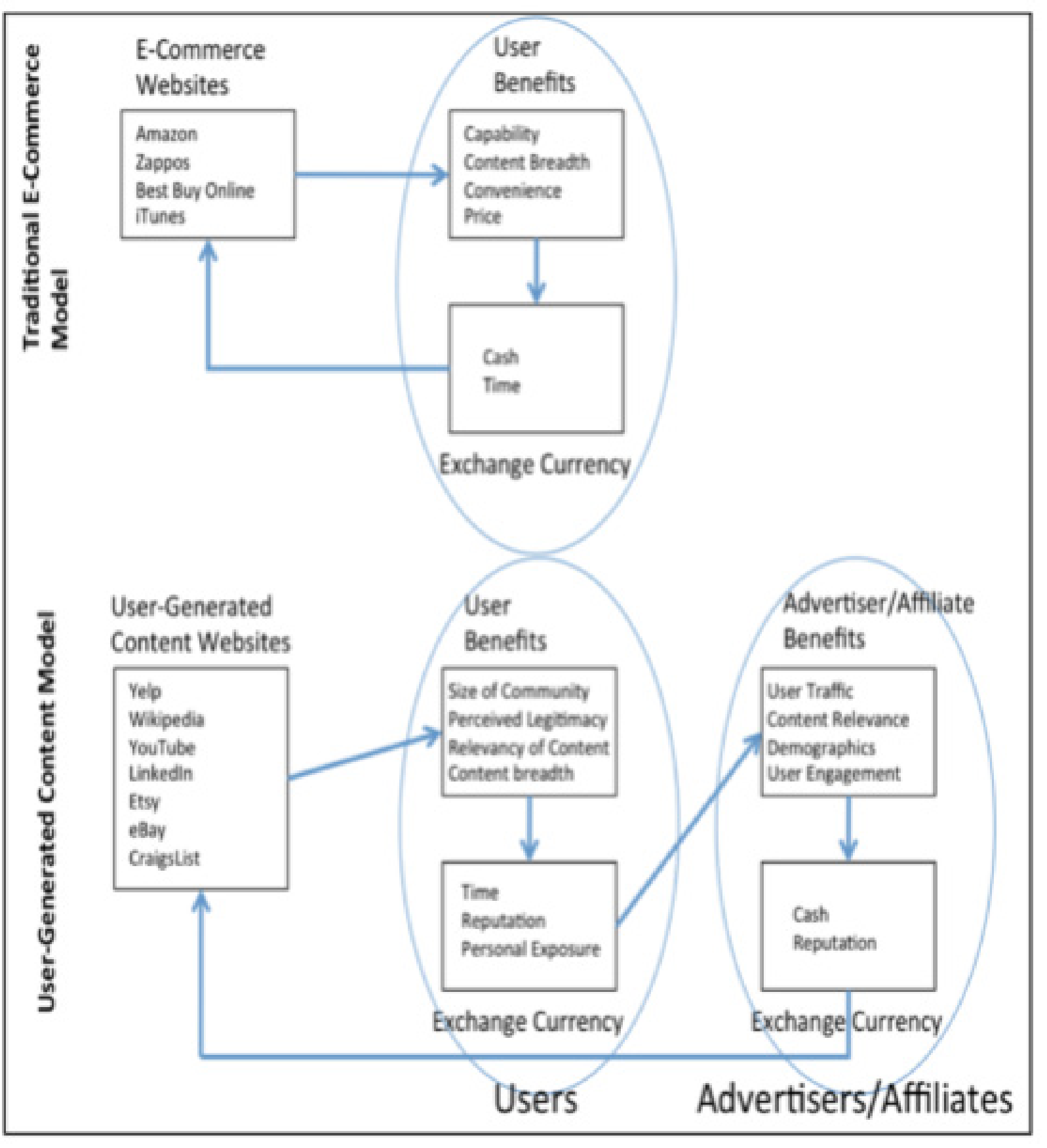

Figure 1: Traditional E-Commerce vs. User-Generated Content Model (Source: Altounian, 2014, p. 4.)

been primarily aimed at comparing the effectiveness of online therapy with face-to-face therapy with a cognitive-behavioral orientation applied to the most prevalent conditions such as depression (De Graaf et al., 2009; Andersson \& Cuijpers, 2009; Andersson et al., 2005) and anxiety (McCrone et al., 2004; Proudfoot et al., 2004). Some have focused on the aspect that is widely considered to be the most influential element to maximize client retention and target outcomes, namely the strength of the client-therapist working alliance (Cook \& Doyle, 2004; Knaevelsrud \& Maercker, 2007; Reynolds, Stiles, \& Grohol, 2007). Although these, and other research reports, are consistently in agreement that online therapy is at least as effective as traditional psychotherapy based on the strength and durability of outcomes, and clients generally reveal similar levels of satisfaction and equally strong therapeutic alliances, knowledge about the specific drivers that determine an online program's effectiveness remains lacking. 
Table 1: Findings of Research on Online Therapy

\begin{tabular}{|l|l|}
\hline \multicolumn{1}{|c|}{ Article } & \multicolumn{1}{|c|}{ Main Findings } \\
De Graaf et al., 2009 & $\begin{array}{l}\text { No significant difference in outcome was found between on- } \\
\text { line, unsupported CBT and a Treatment-as-Usual protocol in } \\
\text { treating depression. }\end{array}$ \\
\hline Andersson \& Cuijpers, 2009 & $\begin{array}{l}\text { A meta-analysis of 12 studies found supported Internet-based } \\
\text { treatments promising to treat depression. }\end{array}$ \\
\hline Andersson et al., 2005 & $\begin{array}{l}\text { Internet-based therapy with minimal therapist contact is effec- } \\
\text { tive to reduce depressive symptoms. }\end{array}$ \\
\hline McCrone et al., 2004 & $\begin{array}{l}\text { Computer-delivered CBT to treat depression is clinically supe- } \\
\text { rior and more cost-effective than the usual treatment. }\end{array}$ \\
\hline Proudfoot et al., 2004 & $\begin{array}{l}\text { Computerized therapy improved symptoms of depression and } \\
\text { anxiety, and did better than usual treatment for more dis- } \\
\text { turbed subjects. }\end{array}$ \\
\hline Cook \& Doyle, 2004 & $\begin{array}{l}\text { Significantly higher means were found on the goal subscale } \\
\text { and composite score of the Working Alliance Inventory in the } \\
\text { online sample compared to a Treatment-as-Usual sample. }\end{array}$ \\
\hline Knaevelsrud \& Maercker, 2007 & $\begin{array}{l}\text { Internet-based therapy proved to be a viable treatment alter- } \\
\text { native for PTSD with large effect sizes and sustained treatment } \\
\text { effects. }\end{array}$ \\
\hline Reynolds, Stiles, \& Grohol, 2007 & $\begin{array}{l}\text { Clients receiving text-based therapy provided similar session } \\
\text { impact and therapeutic alliance ratings compared to face-to- } \\
\text { face clients. }\end{array}$ \\
\hline
\end{tabular}

Although these, and other research reports, are consistently in agreement that online therapy is at least as effective as traditional psychotherapy based on the strength and durability of outcomes, and clients generally reveal similar levels of satisfaction and equally strong therapeutic alliances, knowledge about the specific drivers that determine an online program's effectiveness remains lacking.

\section{User Engagement}

The premise of this research, and other studies that precede it, albeit conducted in other areas of online consumerism, is that there are shared characteristics of a service or consumer website that encourage user engagement, thereby enhancing the users' experience, and increasing their frequency and duration of use, both aspects that determine the success of an online therapy or self-help service. As the most influential of empirical forays into this area, Altounian (2014) found that functionalities of websites that allow visitors more social exchange and penetration that include deepening communication and non-economic exchange increase their commitment levels. In this study, we posit that similar dynamics apply to self-help services, which if proved correct, would mean that facilitated guidance and community participation could influence the process positively.

\section{Mechanical Turk}

The third salient aspect of the current research is the choice of Amazon's Mechanical Turk as a source of participants and delivery mechanism of the research inquiry. Mechanical Turk is a crowdsourcing Internet marketplace that gives businesses and developers as-needed access to human intelligence able to perform required tasks that are currently not within the capability of computers. Tasks are typically fairly menial or repetitive, such as searching and selecting photographs, writing product descriptions, filling in questionnaires, online searches and information collection, data cleaning and verification, and editing and transcribing, and translation of video and audio material. Workers, colloquially known as Turkers, browse through existing jobs and complete selected ones in exchange for a monetary payment set by the employer, which can be as low as one or two dollars per hour. According to a live demographic tracking website, three quarters of currently active Turkers are U.S. residents and 18.75\% from India (see http:// www.mturk-tracker.com).

Although using the MTurk approach is fairly novel in social sciences research, several studies already provide scientific support of its validity to provide a meaningful pool of participants for social and behavioral analyses (Mason \& Suri, 2012; Crump, McDonnell, \& Gureckis, 2013; Rand, 2012). For Mason 


\section{The Protocol}

The objective of the research inquiry is to determine the drivers of user engagement in online self-help programs, using the items and components proposed by Altounian (2014). These are differentiated by participant characteristics that include demographic and personality factors. To achieve these goals, a survey has been designed that includes 149 questions, of which user age group, education and income level, gender, prior experience with mental health services, willingness to participate in online mental health self-help programs, and personality type are variables with which to inform user engagement drivers.

\section{Instruments}

For the purpose of this study, two instruments were used, namely a brief version of the Big Five Personality Inventory, the Big Five Inventory-10 (BFI-10), and Altounian's 4-Factor User Engagement Measurement.

\section{Big Five Inventory-10}

To improve cost- and time-effectiveness, the 44-item Big Five Inventory was abbreviated to 10 items, with research studies establishing that the newly developed brief version, the BFI-10, retained significant levels of reliability and validity, and although effect sizes were lower than those for the full BFI-44, it is still deemed sufficient for research settings with limited time constraints (Rammstedt \& John 2007). The BFI-10 comprises of 10 statements scored on a 5-point Likert scale ranging from "Disagree strongly" to "Agree strongly." With 5 items reverse-scored, five scales of personality are calculated, namely: extraversion, agreeableness, conscientiousness, neuroticism, and openness to experience. Extraversion is characterized by being excitable, social, talkative, assertive, outgoing, and emotionally expressive.

\section{Altounian's 4-Factor User Engagement Measurement}

In his doctoral dissertation (Altounian, 2014) and subsequent studies (Altounian, 2015), David Altounian developed a questionnaire to measure the different drivers of user engagement of service- or content-based websites. He found that the elements could be grouped into four factors, namely: community, site characteristics, content, and user benefit, that impact on the overall user commitment to determine the level and frequency of user engagement and retainment.

The community factor provides an indication of how important community size, contact, and other characteristics are to attract a user to participate on a website. The site characteristics dimension considers aspects such as: the ease of use of a website, functionality, straightforward navigation, privacy, security, access across different devices and browsers, site sharing capability, and credibility among users and peers. The content factor addresses the information provided on the website relating to its stated objectives, expectations, contemporariness, and accuracy, while the user benefit category focuses more on the needs and aspirations of the user in terms of seeking connections and engagement, being able to post and attain badges or award levels, usefulness of the site to help achieve personal or business goals, as well as finding valuable information and being informed. The Altounian user engagement measurement questionnaire comprises of a total of 33 questions rated on a 5-point Likert scale ranging from low to high frequency, low to high agreement, or low to high importance.

\section{Participant Sample}

Participants for this survey were recruited via the Amazon Mechanical Turk platform. The participants knew they would participate in a marketing survey about online self-help. They received a small payment after finishing the survey. Participants were allowed to fill out one survey. The "gig" was only offered to U.S.-based workers.

The online survey included demographic questions, the General Health Questionnaire-12 (GHQ-12), the Adult ADHD Self-Report Scale (ASRS), a brief Big Five personality questionnaire, and Altounian's survey instrument questions (Gosling, Rentfrow, \& Swann 2003; Altounian, 2014). The latter were slightly modified, as the original survey instrument asked questions about a UGC website the participants already knew. Instead, our modified survey asked about "a mental self-help website that you might consider using". Wording in other questions was modified accordingly.

\section{Sample Demographics}

The 184 participants recruited on Amazon Mechanical Turk are all, by design, adults above 18 years and located in the U.S. Figures 2 and 3 illustrate salient demographic characteristics and distribution of the sample, including gender, age group, income and education levels, and whether they have had previous experience with mental health services. The most salient, summarized demographics are as follows: 
- The ratio of male to female participants are almost 3-to-2, with a single person not indicating a gender.

- Just more than half (51\%) of the population sample are between 30 and 49 years old, and 38\% between 19 and 29 years old, with the remaining $11 \% 50$-years and older.

- The largest group, 35\%, indicated education at a Bachelor's degree level, with the second largest group having some college experience, followed by a high school graduate (15\%), an Associate degree $(11 \%)$, and a Graduate professional degree (7\%).

- Regarding income level, the largest group (32\%) reported a $\$ 30,000$ to $\$ 49,000$ annual income, with the group between $\$ 15,000$ to $\$ 29,000$ and the group between $\$ 50,000$ and $\$ 75,000$ trailing at $23 \%$ and $20 \%$ respectively. Eight percent indicated an income less than $\$ 14,999$ annually, and five percent more than $\$ 120,000$, with nine percent coming in between $\$ 75,000$ and $\$ 120,000$, and the remaining three percent preferring not to indicate their income.

- In terms of mental health status, half of the respondents indicated that they had not been diagnosed with any mental health disorder, nor anyone close to them.

- Nearly half (49\%) of the participants have had no prior experience with face-to-face psychotherapy or counseling, while slightly less (40\%) has had therapy themselves, and $11 \%$ indicated that a family member (or members) has had such experience.

\section{Statistical Analysis}

Statistical analyses were performed using R version 3.3.2 and the RStudio graphical user interface.

and Suri, the main benefits of using MTurk to conduct behavioral research is the easy access to a large, stable, and diverse participant pool, the low cost of experiments, and a faster turnaround time between theory and practice. Crump, McDonnell, and Gureckis agreed, adding that the recruitment process can be hastened and dropouts reduced by offering similar payment to someone performing the same task in a lab. They reported reasonably high data quality that compares well to lab results, but pointed to the importance of testing participants' comprehension of the instructions and providing feedback where possible. Although the representativeness of the MTurk participant pool and accuracy of responses are sometimes questioned, Rand reported a higher than $90 \%$ agreement between real and reported data and a similar consistency in a range of demographic variables. However, although many similarities exist between MTurk and traditional samples, there are important differences from broader communities that have to be navigated, including less extraversion and lower self-esteem (Goodman, Cryder, \& Cheema, 2012).

Nevertheless, according to Buhrmester, Kwang, and Gosling (2011), several aspects of the Mechanical Turk setup make it extremely suitable for the participant recruitment and data collection to generate contributions to psychology and other social sciences, in particular: an integrated participant compensation system, a large participant pool, and a streamlined process of study design. These are the qualities that the current study extracted to synthesize data that is valuable for the design and development of online mental health and self-help programs.

\section{Findings}

The correlation coefficients between the individual characteristics (i.e., age, income, education, and personality traits) and the user engagement drives as dependent variables have been determined to their commitment to such a mental health self-help website, and their willingness to try it, as the independent variable. The strength of correlations is reported in Table 2.

The correlation coefficient, $r$, between the commitment that respondents expect to have to a mental health self-help site is related to their likeliness to try such a site, $(0.28, \mathrm{p}<0.05)$. Furthermore, perhaps not surprising, the presence of an official mental health diagnosis for the respondent or a loved one is positively correlated with their willingness to engage with such an online service $(0.1349)$, while their prior experience with face-to-face psychotherapy or counseling does not have a significant impact $(-0.0489)$.

Put in simpler terms, the results in Table 2 indicate those website and personality features that have a positive, negative, or no correlation with the probability that a person will enroll in an online self-help program and their commitment to continue to participate until completion of the program. The larger a number is above zero, the stronger a positive correlation exists, while the smaller a number is below zero, the more significant the negative correlation is. A number at or close to zero indicates little or no correlation between the variables. As such, content and community factors of a self-help website are positively related to a user's commitment to continue to engage and use the service. Extraversion is the 
Table 2: Correlation between User Characteristics, Site Factors, and User Commitment

\begin{tabular}{|l|l|l|}
\hline \multicolumn{1}{|c|}{ Characteristic } & \multicolumn{1}{c|}{ r(Commitment) } & \multicolumn{1}{c|}{ (Likely to enroll) } \\
\hline Site characteristics & $-0.32^{\star}$ & 0.065 \\
\hline Content & $0.72^{\star}$ & $0.21^{\star}$ \\
\hline User benefit & -0.07 & -0.053 \\
\hline Community & $0.24^{\star}$ & 0.079 \\
\hline XTR & $0.21^{\star}$ & 0.078 \\
\hline AGR & 0.084 & 0.042 \\
\hline CON & 0.13 & 0.0025 \\
\hline NEU & $-0.15^{\star}$ & 0.075 \\
\hline OPE & 0.12 & 0.076 \\
\hline
\end{tabular}

$\left({ }^{\star}\right.$ denotes $\left.\mathrm{p}<0.05\right)$

most significant personality feature positively linked to loyalty. The quality and appeal of content is also positively associated with the likelihood that a visitor will enroll in an online program, with no personality trait having a particularly significant positive or negative effect on the decision.

To further inform the statistical correlations between variables, the averages and standard deviations of each of the user engagement drivers and outcome (i.e., commitment) have been determined in the context of the maximum possible score. The results are indicated in Table 3 and Figure 2.

Looking at the data presented in Table 3 and Figure 2 from a non-expert perspective, the horizontal black markers represent the average score for each driver expressed as a percentage of the maximum, with the vertical gray lines indicating one standard deviation, which is a demonstration of the level of variation or dispersion found in the responses of all the participants. That is, a smaller standard deviation implies higher certainty or agreement between the respondents, while a higher standard deviation points to a wider range of responses (i.e., less certainty and agreement). Site characteristics, perceived user benefit, and community features--ranked in this order-have been highlighted as the most significant factors promoting user engagement with the self-help website, while participants felt that content and commitment played a lesser role. These results show that the content and motivation to interact with a website is secondary to features that increase the utility to the user and drive deeper user engagement than undifferentiated and un-nuanced stale subject matter.

A total of $11 \%$ of respondents indicated that they do not consider an online mental health self-help program a useful initiative to pursue and are, therefore, unwilling to consider such a participation. The majority of these participants expressed a concern about the lack of effectiveness of such services and that they are not usually considered credible, although they conceded that online solutions are more convenient, easy to use, and could be available at a lower cost. Of the $58 \%$ that indicated their willingness to consider signing up for an online mental health program (Figure 3), they were most worried about the engagement not being personal enough, but offset this perceived disadvantage by convenience, easeof-use, and advantageous cost factors.

These results give an interesting view of the characteristics and considerations of respondents when it comes to making a decision whether to use an online mental health program, and the factors that will

Table 3: Averages and Standard Deviations of User Engagement Factors

\begin{tabular}{|l|l|l|l|l|}
\hline \multicolumn{1}{|c|}{ User Engagement Factor } & \multicolumn{1}{c|}{ Ave } & \multicolumn{1}{c|}{ SD } & \multicolumn{1}{c|}{ Max } & \multicolumn{1}{c|}{ Ave/Max } \\
\hline Site characteristics & 44.6 & 6.1 & 50 & $89.2 \%$ \\
\hline Content & 19.2 & 5.4 & 35 & $54.9 \%$ \\
\hline User benefit & 31.1 & 4.1 & 40 & $77.8 \%$ \\
\hline Community & 24.8 & 3.4 & 35 & $70.9 \%$ \\
\hline Commitment & 17.5 & 5.0 & 35 & $50.0 \%$ \\
\hline
\end{tabular}




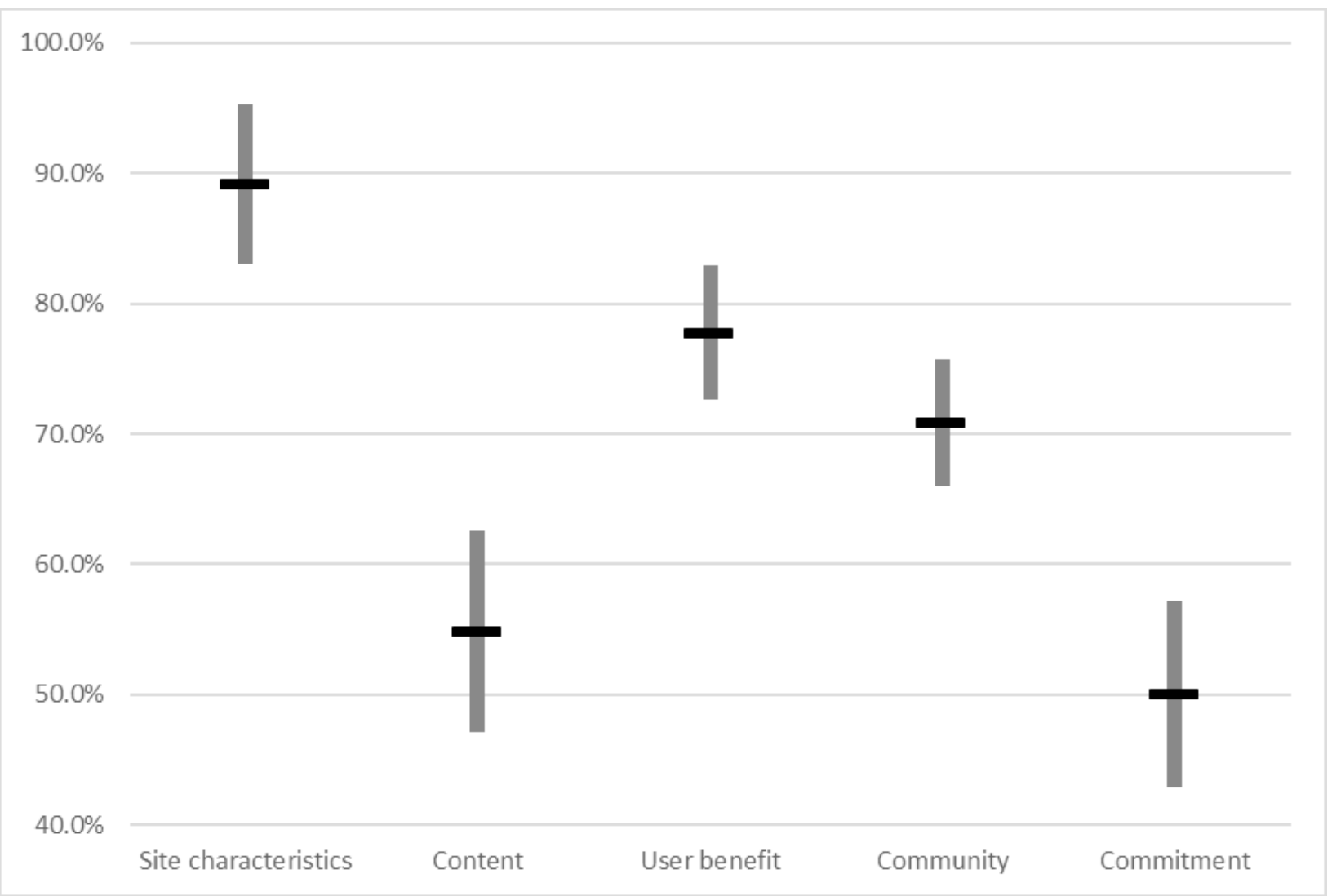

Figure 2: Relative Strength of Engagement Factors

most affect their level of engagement and commitment to such a website. The latter two aspects are the most significant determinants of therapy treatment success, both offline and, presumably, online too.

\section{Discussion}

In this study, the impact of user characteristics (e.g., age, education, and income), personality dimensions (e.g., extraversion, agreeableness, openness, conscientiousness, and neuroticism), and mental health status and therapy experience, and user engagement drivers are determined on their willingness to consider enrolling in an online mental health program and their expectation of commitment to such a program. As somewhat expected, age and education (data not shown) did not appear to have any notable impact on respondents stated willingness nor likeliness to enroll in an online mental health self-help program and neither did it influence their expected commitment to such a program. Their income level had a relatively small, but positive effect on both, with willingness the most prominent with a correlation coefficient of 0.11 . As many respondents mentioned cost as a consideration of participation, such an outcome was anticipated. The presence of an official mental health diagnosis for themselves or a person close to them predicted, with a medi- um correlation (0.13) their willingness to consider online therapy, while their previous experience with face-to-face psychotherapy and counseling did not influence their view in this regard. One can certainly argue that the presence of a diagnosis, is or was associated by symptoms of psychological distress and the need for a suitable intervention, thereby the increased willingness to consider options besides traditional therapy. The fact that their experience with the latter did not appear to have influenced their stance on online therapy is a positive in the sense that it is likely not viewed as competing solutions.

\section{Impact of User Personality Dimensions on Willingness and Commitment}

The impact of the Big Five personality dimensions, namely extraversion, agreeableness, openness, conscientiousness, and neuroticism, on respondents' likeliness to consider enrolling in online therapy and their expected commitment to such a service was determined, and showed that none of the dimensions had any notable correlation with their likeliness to enroll in such a program. However, two of the personality dimensions correlated with their likelihood to pursue and develop a commitment to such an online self-help program. These are extraversion $(r=0.21, p<0.05)$ and neuroticism $(r=-0.15$, 
$\mathrm{p}<0.05)$. This is probably due to the fact that people with high extraversion features have a greater need to engage with others and share experiences, which is true for online as well as offline communities. As those with higher scores on the neuroticism scale may have higher levels of doubt about engaging in online services, their neuroticism may pose a high (and clinically relevant) hurdle to overcome to stimulate their use of online self-help services. On the other hand, one can imagine that once engaged, a certain level of neuroticism may be helpful in staying connected to the program.

\section{Impact of User Engagement Drivers on Willingness and Commitment}

The correlations of Altounian's four drivers of user engagement on a website and as determinants of commitment were calculated to establish which have the greatest impact on potential users' willingness to engage with a mental health website and develop commitment in terms of level of participation, loyalty, retention, and contribution. As mentioned before, motivation to engage and retained commitment are two of the most important factors to predict the outcome and effectiveness of a therapeutic intervention, for both face-to-face and online deliveries. Content $(\mathrm{r}=0.72)$, community factors $(\mathrm{r}=0.24)$ and site characteristics $(\mathrm{r}=-0.32)$ appeared to influence commitment to a website in a statistically significant way.

In this exploratory study we looked at correlations between personality, four factors of user engagement, and commitment to use a website. In a follow-up study we plan to replicate Altounian's path model. Like Altounian, we will use partial least squares structural equation modelling (PLS-SEM) instead of the conventional covariance-based SEM (CB-SEM) because "CB-SEM is more suited to well-researched domains where enough theoretical and substantive knowledge is available, thus CB-SEM can be employed to test the postulated network of relationships among the variables (i.e., test theories). On the other hand, PLSSEM is more appropriate where theory is less developed. They are primarily used to develop theories in exploratory research" (Ravand \& Baghaei 2016).

\section{Conclusions}

In this exploratory study we looked at correlations between personality, four factors of user engagement, and commitment to use a website. We found a strong correlation between content and commitment, and a weaker (and reversed) correlation between site characteristics and commitment. Con- trary to previous research, we found no significant correlation between perceived user benefit and commitment to a UGC website, in this case the use of an online mental health self-help site.

The surveys used may need further tweaking to address the difference between general UGC sites and the specifics of mental health self-help sites. This is likely to strengthen the statistical findings.

\section{References}

Altounian, D. A. (2014). Casual or committed users? Understanding the contributors to user commitment in user-generated content sites. Doctoral dissertation, Oklahoma State University, Stillwater, OK.

Andersson, G., Bergstrom, J., Hollandare, F., Carlbring, P., Kaldo, V., \& Ekselius, L. (2005). Internet-based self-help for depression: Randomized controlled trial. The British Journal of Psychiatry, 187(5), 456-461. DOI: 10.1192/bjp.187.5.456

Andersson, G. A., \& Cuijpers, P. (2009). Internet-based and other computerized psychological treatments for adult depression: A meta-analysis. Cognitive Behaviour Therapy, 38(4), 196-205. DOI: $10.1080 / 16506070903318960$

Buhrmester, M., Kwang, T., \& Gosling, S. D. (2011). Amazon's Mechanical Turk: A new source of inexpensive, yet high-quality, data? Perspectives on Psychological Science, 6(1), 3-5. DOI:

\section{$0.1177 / 1745691610393980$}

Cook, J. E., \& Doyle, C. (2004). Working alliance in online therapy compared to face-to-face therapy: Preliminary results. CyberPsychology \& Behavior, 5(2), 95-105. DOI: 10.1089/109493102753770480

Crump, M. J. C., McDonnell, J. V., \& Gureckis, T. M. (2013). Evaluating Amazon's Mechanical Turk as a tool for experimental behavioral research. PLoS One, 8(3), e57410. DOI: 10.1371/journal. pone. 0057410

De Graaf, L. E., Gerhards, S. A. H., Arntz, A., Riper, H., Metsemakers, J. F. M., Evers, S. M. A. A., Severens, J. L., Widdershoven, G., \& Huibers, M. J. H. (2009). Clinical effectiveness of online computerised cognitive-behavioural therapy without support for depression in primary care: randomised trial. The British Journal of Psychiatry, 195(1), 7380. DOI: 10.1192/bjp.bp.108.054429

Goodman, J. K., Cryder, C. E., \& Cheema, A. (2012). Data collection in a flat world: The strengths and weaknesses of Mechanical Turk samples. Journal 
of Behavioral Decision Making, 26(3), 213-224. DOI: 10.1002/bdm.1753

Gosling, S. D., Rentfrow, P. J., \& Swann, W. B. Jr. (2003). A very brief measure of the big-five personality domains. Journal of Research in Personality, 37(6), 504-528.

Ipeirotis, P. G. (2010). Analyzing the Amazon Mechanical Turk marketplace. XRDS: Crossroads, The ACM Magazine for Students, 17(2), 16-21.

Knaevelsrud, C., \& Maercker, A. (2007). Internet-based treatment for PTSD reduces distress and facilitates the development of a strong therapeutic alliance: A randomized controlled clinical trial. BMC Psychiatry, 7, 13-22. DOI: 10.1186/1471-244X-7-13

Mason, W., \& Suri, S. (2012). Conducting behavioral research on Amazon's Mechanical Turk. Behavioral Research, 44, 1-23. DOI: 10.3758/s13428011-0124-6

McCrone, P., Knapp, M., Proudfoot, J., Ryden, C., Cavanagh, K., Shapiro, D. A., ... Tylee, A. (2004). Cost-effectiveness of computerised cognitive-behavioural therapy for anxiety and depression in primary care: Randomised controlled trial. The British Journal of Psychiatry, 185(1), 55-62. DOI: 10.1192/bjp.185.1.55

Proudfoot, J., Ryden, C., Everitt, B., Shapiro, D. A., Goldberg, D., Mann, A., ... Gray, J. A. (2004). Clinical efficacy of computerised cognitive-behavioural therapy for anxiety and depression in primary care: Randomised controlled trial. The British Journal of Psychiatry, 185(1), 46-54. DOI: 10.1192/bjp.185.1.46
Rammstedt, B., \& John, O. P. (2007). Measuring personality in one minute or less: A 10-item short version of the Big Five Inventory in English and German. Journal of Research in Personality, 41(1), 203-212. DOI: 10.1016/j.jrp.2006.02.001

Rand, D. G. (2012). The promise of Mechanical Turk: How online labor markets can help theorists run behavioral experiments. Journal of Theoretical Biology, 299, 172-179. DOI: 10.1016/j. jtbi.2011.03.004

Ravand, H., \& Baghaei, P. (2016). Partial least squares structural equation modeling with R. Practical Assessment, Research \& Evaluation, 21(11), 1-16.

Reynolds, D. J., Stiles, W. B., \& Grohol, J. M. (2007). An investigation of session impact and alliance in internet based psychotherapy: Preliminary results. Counselling and Psychotherapy Research, 6(3), 164-168. DOI: 10.1080/14733140600853617

Van Dijck, J. (2009). Users like you? Theorizing agency in user-generated content. $\mathrm{Me}$ dia Culture \& Society, 31(1), 41-58. DOI: $10.1177 / 0163443708098245$

\section{Review}

This article was accepted under the constructive peer review option. For futher details, see the descriptions at:

http://mumabusinessreview.org/peer-review-options/

\section{Authors}

Michiel Bosman, MD, PhD is a practitioner-scholar and physician en-
trepreneur with over 15 years of experience in bootstrapping healthcare
related startups. Michiel is the Editor of Open Forest, a provider of on-
line mental health self-help programs and resources. He is also the CEO
of Dokter Bosman, a private outpatient mental health organization in
the Netherlands with almost 300 employees. His research interests are in
Evidence-Based Management and effectivity of online mental health. He
graduated in Medicine from the University of Amsterdam, is a licensed
psychiatrist in the Netherlands, and holds a PhD in Business from Oklaho-
ma State University.

